Скопје, Македонија

\title{
EXTENDING KANNAN AND CHATTERJEA THEOREMS IN 2-BANACH SPACES BY USING SEQUENTIALY CONVERGENT MAPPINGS
}

\author{
Aleksa Malčeski ${ }^{1}$, Alit Ibrahimi $^{2}$, Risto Malčeski ${ }^{3}$
}

\begin{abstract}
S. Gähler ([8]), defined a 2-normed and A. White ([1]) a 2-banach space Further, the contractive mapping in 2-normed space in [4] is defined by in P. K. Hatikrishnan, K. T. Ravindran. In [2] and [10], there are given generalizations of Kannan ([5]) and Chatterjea ([9]) fixed points theorems in 2Banach spaces. In this paper by using sequentially convergent mappings, the results given in [2] and [10] will be generalized in 2-Banach spaces. They might also be considered as generalizations of the results given in [11].
\end{abstract}

\section{INTRODUCTION}

S. Gähler ([8]), 1965 defined a 2-normed space, and White ([1]), 1968, a 2Banach space. 2-Banach spaces are focus of interest of many authors, and certain results can be seen in [6]. Furthermore, analogously as in the normed spaces, P. K. Hatikrishnan and K. T. Ravindran in [4] defined a contractive mapping in 2-normed space as following.

Definition 1 ([4]). Let $(L,\|\cdot, \cdot\|)$ be a real 2-normed space. The mapping $S: L \rightarrow L$ is said to be contraction if $\lambda \in[0,1)$ exists so that $\|S x-S y, z\| \leq \lambda\|x-y, z\|$, for all $x, y, z \in L$, holds true.

Hatikrishnan and Ravindran in [4], proved that a contractive mapping has a unique fixed point in a closed and bounded subset of 2-Banach space. Furthermore, M. Kir and H. Kiziltunc in [2] proved that if $L$ is 2-Banach space and for $\alpha \in\left(0, \frac{1}{2}\right), S: L \rightarrow L$ satisfies one of the following conditions

2010 Mathematics Subject Classification. 46J10, 46J15, 47H10

Key words and phrases. 2-normed spaces, 2-Banach spaces, fixed point, contraction mappings 


$$
\|S x-S y, z\| \leq \alpha(\|x-S x, z\|+\|y-S y, z\|), \text { for all } x, y, z \in L
$$

or

$$
\|S x-S y, z\| \leq \alpha(\|x-S y, z\|+\|y-S x, z\|), \text { for all } x, y, z \in L
$$

then, $S$ has a unique fixed point in $L$. The case where the condition (1) is satisfied is actually a generalization of Kannan's Theorem and the case where the condition (2) is satisfied is a generalization of Chatterjea's Theorem. Furthermore, the generalizations of M. Kir and H. Kiziltunc results and their consequences are reviewed in [10]. Next, we will make a generalization of the above mentioned results, by using sequentially convergent mappings, defined as following.

Definition 2. Let $(L,\|\cdot, \cdot\|)$ be a 2-normed space. A mapping $T: L \rightarrow L$ is said to be sequentially convergent if for each sequence $\left\{y_{n}\right\}$ the following condition is satisfied:

If $\left\{T y_{n}\right\}$ converges, then $\left\{y_{n}\right\}$ is also converges.

\section{EXTENSIONS OF KANNAN AND CHATTERJEA THEOREMS}

Theorem 1. Let $(L,\|\cdot, \cdot\|)$ be a 2 -Banach space, $S: L \rightarrow L$ and the mapping $T: L \rightarrow L$ is continuous, injection and sequentially convergent. If $\alpha>0, \gamma \geq 0$, are such that $2 \alpha+\gamma<1$, for all $x, y, z \in L$, and

$$
\|T S x-T S y, z\| \leq \alpha(\|T x-T S x, z\|+\|T y-T S y, z\|)+\gamma\|T x-T y, z\|,
$$

then, $S$ has a unique fixed point and for each $x_{0} \in L$ the sequence $\left\{S^{n} x_{0}\right\}$ converges to the above fixed point.

Proof. Let $x_{0}$ be any point in $L$ and let the sequence $\left\{x_{n}\right\}$ be defined as $x_{n+1}=S x_{n}, n=0,1,2, \ldots$. The inequality (3) and the definition of $\left\{x_{n}\right\}$ imply that

$$
\left\|T x_{n}-T x_{n+1}, z\right\| \leq \alpha\left(\left\|T x_{n-1}-T x_{n}, z\right\|+\left\|T x_{n}-T x_{n+1}, z\right\|\right)+\gamma\left\|T x_{n}-T x_{n-1}, z\right\|
$$

This holds true for each $n=0,1,2,3, \ldots$ and for each $z \in L$. So, the inequality above implies

$$
\left\|T x_{n}-T x_{n+1}, z\right\| \leq \lambda^{n}\left\|T x_{0}-T x_{1}, z\right\|
$$

for each $n=0,1,2,3, \ldots$ and each $z \in L, \quad 0<\lambda=\frac{\alpha+\gamma}{1-\alpha}<1$. Furthermore, (4) implies that for all $m, n \in \mathbf{N}, n>m$ and for each $z \in L$ 


$$
\left\|T x_{n}-T x_{m}, z\right\| \leq \frac{\lambda^{m}}{1-\lambda}\left\|T x_{0}-T x_{1}, z\right\|
$$

holds true. The sequence $\left\{T x_{n}\right\}$ is Caushy and $L$ is a 2-Banach space. So, the sequence $\left\{T x_{n}\right\}$ is convergent. Furthermore, the mapping $T: L \rightarrow L$ is sequentially convergent, so the sequence $\left\{x_{n}\right\}$ is convergent, i.e. it exists $u \in L$ so that $\lim _{n \rightarrow \infty} x_{n}=u$.

Since $T$ is continuous, $\lim _{n \rightarrow \infty} T x_{n}=T u$ and

$$
\begin{aligned}
\| T S u-T u, z & \|\leq\| S u-T S^{n} x_{0}, z\|+\| T S^{n} x_{0}-T S^{n+1} x_{0}, z\|+\| T S^{n+1} x_{0}-T u, z \| \\
\leq & \alpha\left(\|T u-T S u, z\|+\left\|T S^{n-1} x_{0}-T S^{n} x_{0}, z\right\|\right)+\gamma\left\|T u-T S^{n-1} x_{0}, z\right\| \\
& +\left\|T S^{n} x_{0}-T S^{n+1} x_{0}, z\right\|+\left\|T S^{n+1} x_{0}-T u, z\right\| \\
= & \alpha\left(\|T u-T S u, z\|+k\left\|T x_{n-1}-T x_{n}, z\right\|\right)+\gamma\left\|T u-T x_{n-1}, z\right\| \\
& +\left\|T x_{n}-T x_{n+1}, z\right\|+\left\|T x_{n+1}-T u, z\right\| .
\end{aligned}
$$

holds true for each $n=0,1,2,3, \ldots$ and each $z \in L$.

For $n \rightarrow \infty$, the continuous of $T$ and the properties of 2-norm imply that $\|T S u-T u, z\| \leq \alpha\|T S u-T u, z\|$ holds true for each $z \in L$. Since, $\alpha<1$, this implies that $\|T S u-T u, z\|=0$, for each $z \in L$, i.e. $T S u=T u$. Finally, $T$ is injection, so, $S u=u$, and $S$ has a fixed point.

Let $u, v \in X$ be fixed points of $S$, i.e. $S u=u$ and $S v=v$. Then, (3) implies that $\|T u-T v, z\|=\|T S u-T S v, z\| \leq \alpha(\|T u-T S u, z\|+\|T v-T S v, z\|)+\gamma\|T u-T v, z\|$ holds true for each $z \in L$. Since $\gamma<1$, we get that $\|T u-T v, z\|=0$, for each $z \in L$, i.e. $T u=T v$. Since $T$ is an injection, $u=v$, so $T$ has a unique fixed point. Finally, the arbitrarily of $x_{0} \in L$ implies that for each $x_{0} \in L$ the sequence $\left\{S^{n} x_{0}\right\}$ converges to the unique fixed point of $S$.

Consequence 1. Let $(L,\|\cdot \cdot\|)$ be a 2-Banach space, $S: L \rightarrow L$ and the mapping $T: L \rightarrow L$ be continuous, injection and sequentially convergent. If $\lambda \in(0,1)$ and

$$
\|T S x-T S y, z\| \leq \lambda \sqrt[3]{\|T x-T S x, z\| \cdot\|T y-T S y, z\| \cdot\|T x-T y, z\|}
$$

holds true for all $x, y, z \in L$, then $S$ has a unique fixed point.

Proof. For $\alpha=\gamma=\frac{\lambda}{3}$, the arithmetic-geometric inequality mean and the theorem 1 , directly imply the statement.. 
Consequence 2. Let $(L,\|\cdot, \cdot\|)$ be a 2-Banach space, $S: L \rightarrow L$ and the mapping $T: L \rightarrow L$ be continuous, injection and sequentially convergent. If there exist $\alpha>0$, and $\gamma \geq 0$, so that $2 \alpha+\gamma<1$ and

$$
\|T S x-T S y, z\| \leq \alpha \frac{\|T x-T S x, z\|^{2}+\|T y-T S y, z\|^{2}}{\|T x-T S x, z\|+\|T y-T S y, z\|}+\gamma\|T x-T y, z\|,
$$

holds true for all $x, y, z \in L$, then $S$ has a unique fixed point.

Proof. The inequality sated in the condition implies (3), and the required statement is directly implied by the theorem 1 .

Consequence 3. Let $(L,\|\cdot, \cdot\|)$ be a 2-Banach space, $S: L \rightarrow L$ and the mapping $T: L \rightarrow L$ be continuous, injection and sequentially convergent. If $\alpha \in\left(0, \frac{1}{2}\right)$ and

$$
\|T S x-T S y, z\| \leq \alpha(\|T x-T S x, z\|+\|T y-T S y, z\|)
$$

holds true for all $x, y, z \in L$, then $S$ has a unique fixed point.

Proof. For $\gamma=0$, and applying the theorem 1, we get the required statement.

Remark 1. The theorem 1 and the consequences 1 and 2 , for $T x=x$, imply the validity of the theorem 1 and the consequences 1 and 2, [10], and the consequence 3 , imply the validity of the theorem $1,[2]$.

Theorem 2. Let $(L,\|\cdot, \cdot\|)$ be a 2-Banach space, $S: L \rightarrow L$ and the mapping $T: L \rightarrow L$ be continuous, injection and sequentially convergent. If $\alpha>0, \gamma \geq 0$, such that $2 \alpha+\gamma<1$ and

$$
\|T S x-T S y, z\| \leq \alpha(\|T x-T S y, z\|+\|T y-T S x, z\|)+\gamma\|T x-T y, z\|
$$

holds true for all $x, y, z \in L$, then $S$ has a unique fixed point and for each $x_{0} \in X$ the sequence $\left\{S^{n} x_{0}\right\}$ converges to the above fixed point.

Proof. Let $x_{0}$ be any point in $L$ and the sequence $\left\{x_{n}\right\}$ be defined as the following $x_{n+1}=S x_{n}, n=0,1,2,3, \ldots$. The inequality (5) implies that

$$
\begin{aligned}
\| T x_{n+1}-T x_{n}, z & \left\|=T S x_{n}-T S x_{n-1}, z\right\| \\
& \leq \alpha\left(\left\|T x_{n}-T S x_{n-1}, z\right\|+\left\|T x_{n-1}-T S x_{n}, z\right\|\right)+\gamma\left\|T x_{n}-T x_{n-1}, z\right\| \\
& =\alpha\left\|T x_{n+1}-T x_{n-1}, z\right\|+\gamma\left\|T x_{n}-T x_{n-1}, z\right\| \\
& \leq \alpha\left(\left\|T x_{n+1}-T x_{n}, z\right\|+\left\|T x_{n}-T x_{n-1}, z\right\|\right)+\gamma\left\|T x_{n}-T x_{n-1}, z\right\|
\end{aligned}
$$

holds true for each $n=0,1,2,3, \ldots$ and each $z \in L$. The previous inequality implies that 


$$
\left\|T x_{n}-T x_{n+1}, z\right\| \leq \lambda^{n}\left\|T x_{0}-T x_{1}, z\right\|
$$

holds true for each $n=0,1,2,3, \ldots$ and each $z \in L$, for $0<\lambda=\frac{\alpha+\gamma}{1-\alpha}<1$. Furthermore, by using (6), analogously as in the theorem 1is is proven that the sequence $\left\{x_{n}\right\}$ is convergent, i.e. it exists $u \in L$ so that $\lim _{n \rightarrow \infty} x_{n}=u$ and $\lim _{n \rightarrow \infty} T x_{n}=T u$. From the inequality (5), for each $n=0,1,2,3, \ldots$ and each $z \in L$, $n \rightarrow \infty$

$$
\begin{aligned}
& \|T S u-T u, z\| \leq\left\|T S u-T S^{n} x_{0}, z\right\|+\left\|T S^{n} x_{0}-T S^{n+1} x_{0}, z\right\|+\left\|T S^{n+1} x_{0}-T u, z\right\| \\
& \leq \alpha\left(\left\|T u-T S^{n} x_{0}, z\right\|+\left\|T S^{n-1} x_{0}-T S u, z\right\|\right)+\gamma\left\|T u-T S^{n-1} x_{0}, z\right\| \\
& +\left\|T S^{n} x_{0}-T S^{n+1} x_{0}, z\right\|+\left\|T S^{n+1} x_{0}-T u, z\right\| \\
& =\alpha\left(\left\|T u-T x_{n}, z\right\|+\left\|T x_{n-1}-T S u, z\right\|\right)+\gamma\left\|T u-T x_{n-1}, z\right\| \\
& +\left\|T x_{n}-T x_{n+1}, z\right\|+\left\|T x_{n+1}-T u, z\right\| .
\end{aligned}
$$

holds true. Analogously, as in the theorem 1, we come to the conclusion that $S u=u$, i.e. $u$ is a fixed point of $S$. Finally, if $v$ is another fixed point of $S$, the inequality (5) implies that

$$
\|T u-T u, z\| \leq(2 \alpha+\gamma)\|T u-T v, z\|,
$$

holds true for each $z \in L$. This implies that $u=v$.

Consequence 4. Let $(L,\|\cdot, \cdot\|)$ be a 2-Banach space, $S: L \rightarrow L$ the mapping $T: L \rightarrow L$ be continuous, injection and sequentially convergent. If $\lambda \in(0,1)$ and

$$
\|T S x-T S y, z\| \leq \lambda \sqrt[3]{\|T x-T S y, z\| \cdot\|T y-T S x, z\| \cdot\|T x-T y, z\|}
$$

for all $x, y, z \in L$, then $S$ has a unique fixed point.

Proof. For $\alpha=\gamma=\frac{\lambda}{3}$, the arithmetic-geometric inequality mean and theorem 2 imply the validity of the statement above.

Consequence 5. Let $(L,\|\cdot \cdot\|)$ be a 2-Banach space, $S: L \rightarrow L$ and the mapping $T: L \rightarrow L$ be continuous, injection and sequentially convergent. If there exist $\alpha>0, \gamma \geq 0$ such that $2 \alpha+\gamma<1$ and

$$
\|T S x-T S y, z\| \leq \alpha \frac{\|T x-T S y, z\|^{2}+\|T y-T S x, z\|^{2}}{\|T x-T S y, z\|+\|T y-T S x, z\|}+\gamma\|T x-T y, z\|,
$$

for all $x, y, z \in L$, then $S$ has a unique fixed point.

Proof. The inequality given in the condition implies the inequality (5). Finally, the required statement is directly implied by the theorem 2 . 
Consequence 6. Let $(L,\|\cdot, \cdot\|)$ be a 2-Banach space, $S: L \rightarrow L$ and the mapping $T: L \rightarrow L$ be continuous, injection and sequentially convergent. If $\alpha \in\left(0, \frac{1}{2}\right)$ and

$$
\|T S x-T S y, z\| \leq \alpha(\|T x-T S y, z\|+\|T y-T S x, z\|)
$$

holds true for all $x, y, z \in L$, then $S$ has a unique fixed point.

Proof. For $\gamma=0$, and applying the theorem 2, we get the required statement.

Remark 2. The theorem 2 and the consequences 4 and 5, for $T x=x$, imply the validity of the theorem 2 and the consequences 3 and 4, [10], also the consequence 6 confirm the validity of the theorem 2, [2].

\section{EXTENSIONS OF KOPARDE-WAGHMODE THEOREM}

Theorem 3. Let $(L,\|\cdot, \cdot\|)$ be a 2 -Banach space, $S: L \rightarrow L$ and the mapping $T: L \rightarrow L$ be continuous, injection and sequentially convergent. If $\alpha>0, \gamma \geq 0$, such that $2 \alpha+\gamma<1$ and

$$
\|T S x-T S y, z\|^{2} \leq \alpha\left(\|T x-T S x, z\|^{2}+\|T y-T S y, z\|^{2}\right)+\gamma\|T x-T y, z\|^{2}
$$

holds true for all $x, y, z \in L$, then $S$ has a unique fixed point and for each $x_{0} \in X$ the sequence $\left\{S^{n} x_{0}\right\}$ converges to the above fixed point.

Proof. Let $x_{0}$ be any point in $L$ and let the sequence $\left\{x_{n}\right\}$ be defined as $x_{n+1}=S x_{n}, n=0,1,2,3, \ldots$. The inequality (7) implies the following

$$
\begin{aligned}
\left\|T x_{n+1}-T x_{n}, z\right\|^{2} & =\left\|T S x_{n}-T S x_{n-1}, z\right\|^{2} \\
& \leq \alpha\left(\left\|T x_{n}-T S x_{n}, z\right\|^{2}+\left\|T x_{n-1}-T S x_{n-1}, z\right\|^{2}\right)+\gamma\left\|T x_{n}-T x_{n-1}, z\right\|^{2} \\
& =\alpha\left(\left\|T x_{n}-T x_{n+1}, z\right\|^{2}+\left\|T x_{n-1}-T x_{n}, z\right\|^{2}\right)+\gamma\left\|T x_{n}-T x_{n-1}, z\right\|^{2},
\end{aligned}
$$

for each $n=0,1,2,3, \ldots$. The previous inequality and the condition given in the theorem, imply that for $\lambda=\sqrt{\frac{\alpha+\gamma}{1-\alpha}}<1$ the following holds true

$$
\left\|T x_{n+1}-T x_{n}, z\right\|^{2} \leq \lambda\left\|T x_{n}-T x_{n-1}, z\right\|^{2},
$$

for each $n=0,1, \ldots$ and for each $z \in L$. Furthermore, analogously as the theorem 1 and 2 we get that the sequence $\left\{x_{n}\right\}$ is convergent, i.e. there is $u \in L$ such that $\lim _{n \rightarrow \infty} x_{n}=u$ and also the continuous of $T$ imply that $\lim _{n \rightarrow \infty} T x_{n}=T u$. 
We will prove that $u$ is a fixed point of $S$. Namely, by using the inequality (7), it is easy to prov that $\|T S u-T u, z\| \leq \sqrt{\alpha}\|T S u-T u, z\|$, holds true for each $z \in L$. Since $\sqrt{\alpha}<1$, we get that $\|T S u-T u, z\|=0$, holds true for each $z \in L$. Analogously as the proof of the theorem 1, we get that $u$ is a fixed point of $S$. If $v$ is one other fixed point of $S$, then (7) implies $\|T u-T v, z\| \leq \gamma\|T u-T v, z\|$ , for each $z \in L$. Thus, analogously to the proof of theorem $1, u=v$.

Consequence 7. Let $(L,\|\cdot, \cdot\|)$ be a 2-Banach space, $S: L \rightarrow L$ and the mapping $T: L \rightarrow L$ be continuous, injection and sequentially convergent. If $\alpha \in\left(0, \frac{1}{2}\right)$ and

$$
\|T S x-T S y, z\|^{2} \leq \alpha\left(\|T x-T S y, z\|^{2}+\|T y-T S x, z\|^{2}\right),
$$

for all $x, y, z \in L$, then $S$ has a unique fixed point.

Proof. For $\gamma=0$ in the theorem 3, we get the required statement.

Remark 3. For $T x=x$, the theorem 3 and the consequence 7 , we get the validity of the theorem 3 and the consequence 5, [10].

\section{Conclusion}

In our previus observations by using the sequential convergent mapping we generalized several results about fixed point in 2-Banach space ([10]). Naturally, we wonder if Kannan, Chatterjea and Koparde-Waghmode theorems about common fixed point of mappings defined in 2-Banach spaces might be generalized by using sequentially continuous mapping?

\section{CONFLICT OF INTEREST}

No conflict of interest was declared by the authors.

\section{AUTHOR'S CONTRIBUTIONS}

All authors contributed equally and significantly to writing this paper. All authors read and approved the final manuscript. 
References

[1] A. White, 2-Banach Spaces, Math. Nachr. Vol. 42 (1969), 43-60

[2] M. Kir, H. Kiziltunc, Some New Fixed Point Theorems in 2-Normed Spaces, Int. Journal of Math. Analysis, Vol. 7 No. 58 (2013), 2885-2890

[3] P. Chouhan, N. Malviya, Fixed Points of Expansive Type Mappings in 2Banach Spaces, International Juornal of Analysis and Applications, Vol. 3 No. 1 (2013), 60-67

[4] P. K. Hatikrishnan, K. T. Ravindran, Some Properties of Accretive Operators in Linear 2-Normed Spaces, International Mathematical Forum, Vol. 6 No. 59 (2011), 2941-2847

[5] R. Kannan, Some results on fixed points, Bull. Calc. Math. Soc. Vol. 60 No. 1, (1968), 71-77

[6] R. Malčeski, K. Anevska, About the 2-Banach spaces, International Journal of Modern Engineering Research (IJMER), Vol. 4 Iss. 5 (2014), 28-32

[7]S. Banach, Sur les operations dans les ensembles abstraits et leur application aux equations intégrales, Fund. Math. 2 (1922), 133-181

[8] S. Gähler, Lineare 2-normierte Räume, Math. Nachr. 28 (1965), 1-42

[9] S. K. Chatterjea, Fixed point theorems, C. R. Acad. Bulgare Sci., Vol. 25 No. 6 (1972), 727-730

[10] R. Malčeski, A. Ibrahimi, Contraction Mappings and Fixed Point in 2Banach Spaces, IJSIMR, Vol. 4, Issue 4, pp. 34-43

[11] A. Malčeski, S. Malčeski, K. Anevska, R. Malčeski, New Extension of Kannan and Chatterjea Fixed Point Theorems on Complete Metric Spaces, British Journal of Mathematics \& Computer Science, Vol. 17, No. 2, (2016), 1-10

[12] S. Moradi, D. Alimohammadi, New Extensions of Kannan Fixed Theorem on Complete Metric and Generalized Metric Spaces, Int. Journal of Math. Analysis, Vol. 5 No. 47 (2011), 2313-2320

\footnotetext{
${ }^{1)}$ Faculty of Mechanical Engineering, Ss. Cyril and Methodius University, Skopje, Macedonia E-mail address: aleksa.malceski@gmail.com

2) University of Tetovo, Macedonia E-mail address: ibraimia@yahoo.com

${ }^{3)}$ Faculty of informatics, FON University, Skopje, Macedonia E-mail address: risto.malceski@gmail.com
} 